\title{
A Case of Epithelioid Blue Nevus
}

\author{
Chung Hun Lee $\cdot$ Hye Sook Min ${ }^{1}$ Eon Sub Park · Kye Yong Song \\ Department of Pathology, Chung-Ang University College of Medicine; 'Department of Pathology, Seoul National University College of Medicine, Seoul, Korea
}

Although epithelioid blue nevus (EBN) is a rare variant of blue nevus that was first reported in patients with Carney complex, which encompasses cardiac and cutaneous myxomas, spotty skin pigmentation, endocrine overactivity, and psammomatous melanotic schwannomas, ${ }^{1}$ it has been also documented in the absence of such clinical features. ${ }^{2}$ Histological characteristics of EBN are variably pigmented, large epithelioid melanocytes with vesicular nuclei and prominent eosinophilic nucleoli and a small number of melanophages that are sometimes associated with a few pigmented, spindle, and dendritic cells. ${ }^{3}$ EBN is a diagnostically challenging entity because of its rarity and histological overlap with other pigmented melanocytic lesions, such as cellular blue nevus, malignant blue nevus, and the so-called animal-type malignant melanoma. Because it is very uncommon and no cases have been recorded in the Korean literature, this Korean case is reported.

\section{CASE REPORT}

A 21-year-old woman presented with a bluish nodule that had been slowly growing for approximately 10 years on the dorsum of the right foot (Fig. 1A). The lesion was dome-shaped and measured $1.0 \mathrm{~cm}$ in diameter. There were no clinical features of Carney complex. The lesion was completely excised.

The histological examination showed a relatively well circumscribed, round nodule with heavy pigmentation in the reticular dermis with extension into the subcutaneous adipose tissue (Fig.

\section{Corresponding Author}

Kye Yong Song, M.D.

Department of Pathology, Chung-Ang University Hospital, 102 Heukseok-ro,

Dongjak-gu, Seoul 156-755, Korea

Tel: +82-2-6299-3167, Fax: +82-2-6293-5630, E-mail: sky@cau.ac.kr

Received: January 15, 2014 Revised: February 27, 2014

Accepted: March 14, 2014
1B). The tumor was composed of heavily pigmented, epithelioid melanocytes with vesicular nuclei and prominent nucleoli (Fig. 1C, D). Neither mitotic figures nor infiltrative growth patterns were noted. Melanin pigmentation was variable in the epithelioid cells. A small number of fusiform-shaped melanocytes and dendritic spindled melanocytes were also found among the pigmented epithelioid melanocytes. No junctional or dermal banal nevus component was present, and the papillary dermis was spared. The deepest boundary of the tumor was a pushing border with mild fibrosis.

Immunohistochemical findings included pigmented epithelioid cells positive for S-100 protein (Fig. 2A) and Melan A (Fig. 2B), while only a few cells were reactive for HMB45 (Fig. 2C). A few CD68-positive melanophages were noted (Fig. 2D). These results indicate that almost all pigmented epithelioid cells were epithelioid melanocytes. Ki-67-labeled nuclei were very rare. All of these findings were consistent with EBN.

\section{DISCUSSION}

Blue nevus is a tumor of dermal dendritic melanocytic origin, and common blue nevus and cellular blue nevus are the most common variants. ${ }^{3} \mathrm{EBN}$ is another variant of blue nevus that arises in the background of Carney complex ${ }^{1}$ or may occur sporadically. ${ }^{2}$ The main tumor cells of common blue nevus are elongated, dendritic melanocytes with reactive melanophages and only a few epithelioid melanocytes. In contrast, the major component of EBN is variably pigmented, epithelioid melanocytes with a few melanophages and only a few dendritic melanocytes. Although epithelioid melanocytes appear similar to melanophages and epithelioid neural cells, the nature of epithelioid melanocytic cells can be demonstrated by immunohistochemistry for $\mathrm{S} 100$ protein, Melan-A, HMB 45, and CD68. The 

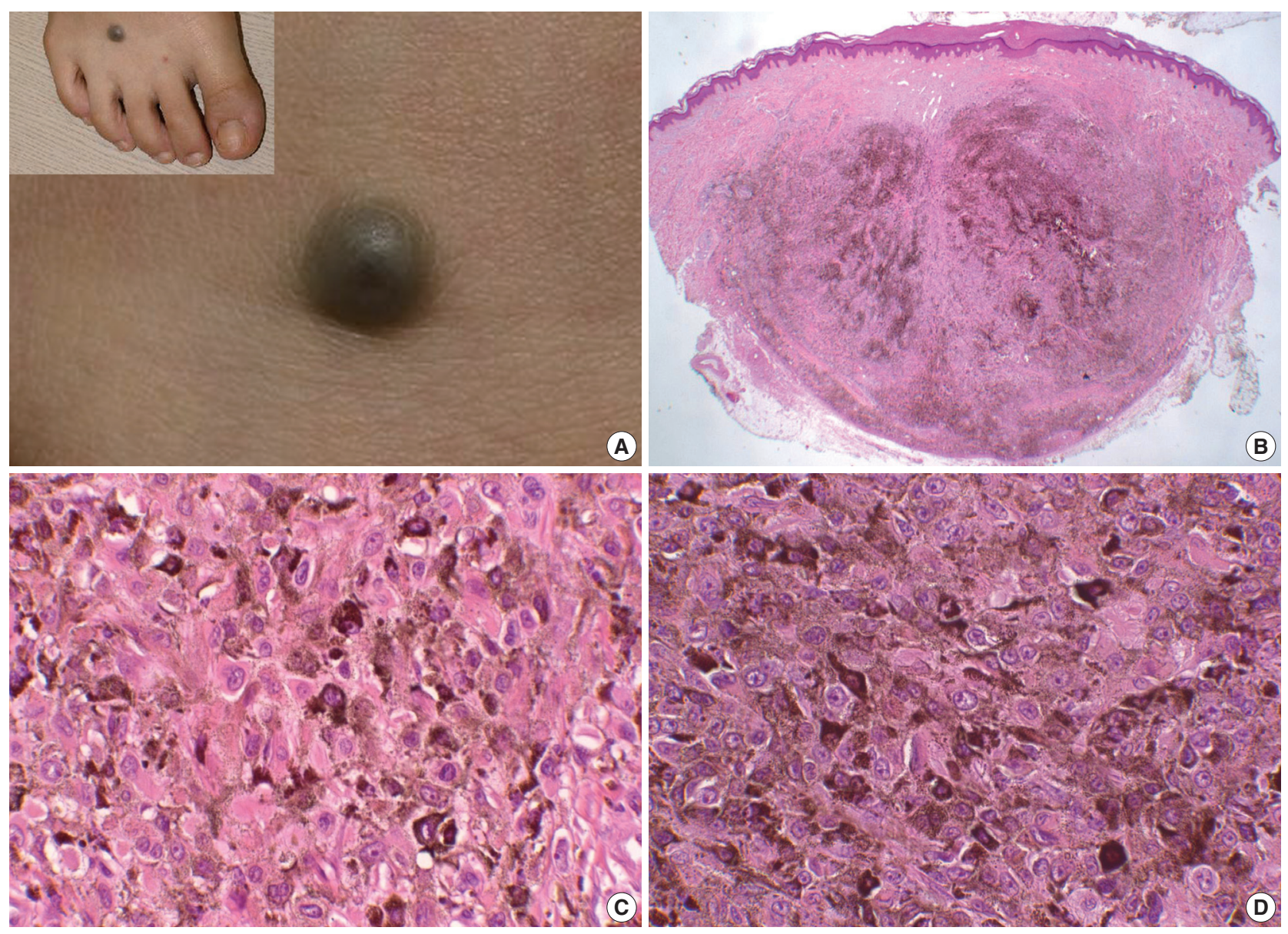

Fig. 1. (A) A case of epithelioid blue nevus. A relatively well defined, round and bluish nodule is developed on the dorsum of right foot (A, inset in A). (B) Excised tumor is heavily pigmented and relatively well demarcated in dermis with expansion to the subcutis. (C) High power view shows variably pigmented epitheloid melanocytes with eosinophilic cytoplasm. (C, D) Some epithelioid melanocytes show prominent nucleoli, but no mitotic figure is noted.

differential diagnoses of EBN include cellular blue nevus, epithelioid schwannoma, pigmented epithelioid Spitz nevus, malignant blue nevus, malignant melanoma, metastatic melano$\mathrm{ma}$, tumoral melanosis, and pigment synthesizing melanoma. Clinicopathological correlation is required for differential diagnosis of these pigmented epithelioid tumors.

Malignant melanoma and malignant blue nevus exhibit epithelioid cells with malignant features, such as infiltrative growth, necrosis, mitoses, pleomorphism, and hyperchromasia. ${ }^{4}$ Metastatic melanoma can mimic blue nevi in that it may be composed of pigmented epithelioid cells in an entirely intradermal location. However, such a melanoma displays hyperchromatic nuclei and frequent mitosis. ${ }^{5}$

Tumoral melanosis, a regressed stage of a melanocytic lesion, such as malignant melanoma, should also be differentiated. ${ }^{6}$ The pigmented cells of tumoral melanosis are pigment-laden macrophages that are negative for Melan A and S-100 protein.
Only a few reported cases of heavily pigmented epithelioid Spitz nevus are found in the English literature. These nevi and EBN share pigmented epithelioid melanocytes of the dermis, but the former show a junctional component. ${ }^{7}$ Although cases of EBN with junctional nests have been described, ${ }^{8}$ they are considered combined nevi rather than pure EBN. On the contrary, this EBN case showed no evidence of banal nevus cell nests.

Pigment synthesizing melanoma, also called animal-type melanoma is a variant of human melanoma that resembles that found in elderly grey horses. ${ }^{9}$ It is considered a low-grade melanoma with histological features of poor demarcation, heavily pigmented epithelioid cells, and short spindle-shaped melanocytes with hyperchromatic nuclei and prominent nucleoli, junctional components, melanophages, and extension to deep dermis, but no dendritic melanocytes. ${ }^{10}$ Even though epithelioid melanocytes predominate in both EBN and animal-type melanoma, the main difference is the growth pattern. While the former is expansile, 

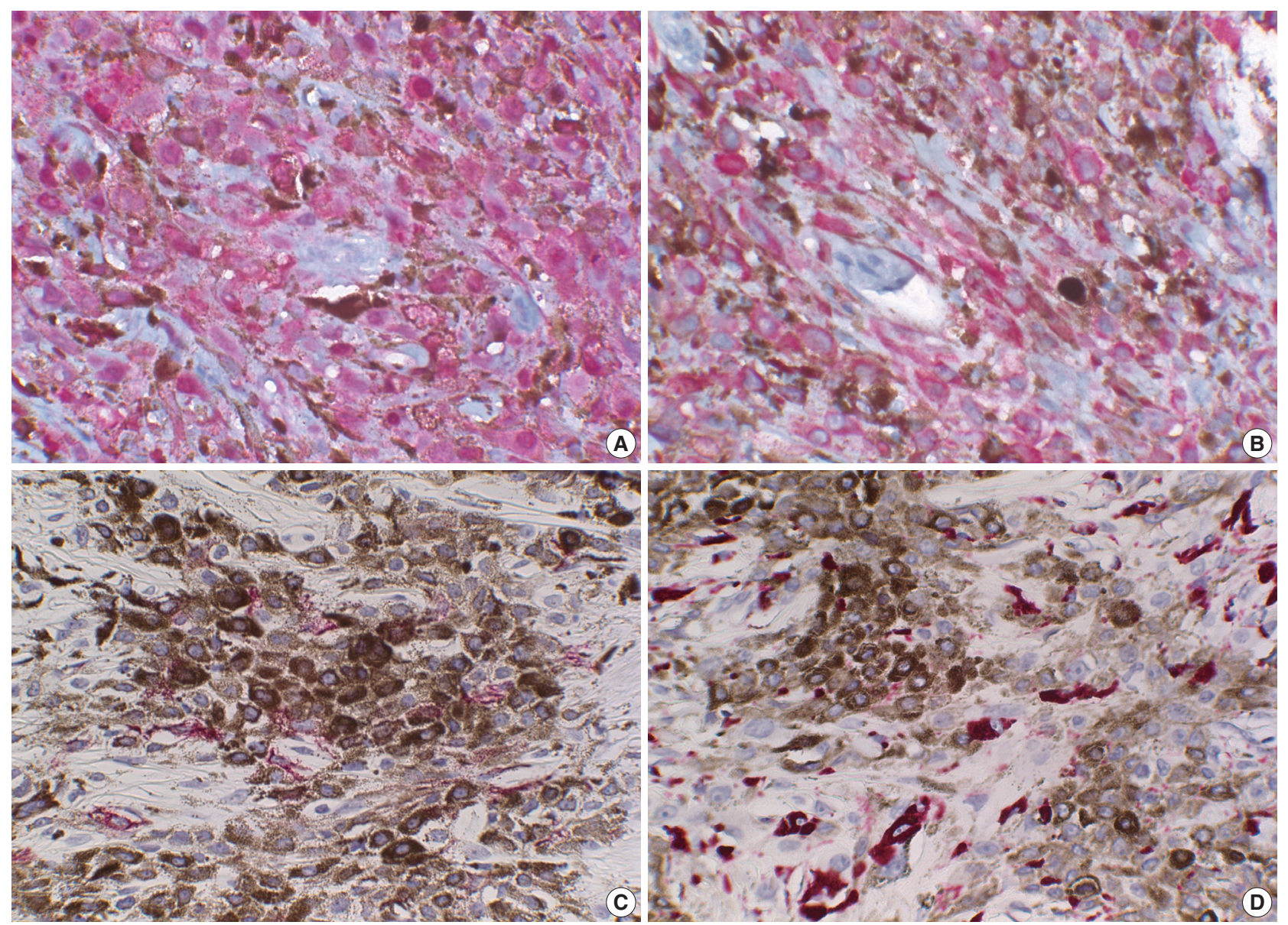

Fig. 2. Immunohistochemical stains of epithelioid melanocytes. S-100 protein (A) and Melan-A (B) are positive in nearly all epithelioid cells, and human melanoma black 45 is positive in a few epithelioid cells (C). (D) CD68 is positive in a few melanophages.

the latter is infiltrative. In addition, the cells of animal-type melanoma have malignant features. ${ }^{6,10}$ Nevertheless, differential diagnosis between them is sometimes difficult because both demonstrate low mitotic count, absent or non-brisk tumor-infiltrating lymphocytes, and an absence of vascular or perineural invasion. ${ }^{6,10}$ Thus, the term "pigmented epithelioid melanocytoma" is also proposed, which reflects that both of these two entities are within the same disease spectrum. ${ }^{6}$ Furthermore, sentinel lymph node biopsy is recommended as a diagnostic procedure to discriminate the two entities. ${ }^{6}$

The term "pigmented epithelioid melanocytoma" may give a misleading implication that this tumor is benign, when in fact it is a low-grade malignant melanoma. Therefore, we suggest that benign epithelioid melanocytic EBN and malignant lesions be named "pigmented epithelioid melanoma" rather than pigmented epithelioid melanocytoma or animal-type melanoma. Because there is no clinical or histological evidence of a malignant nature, the present case is a benign pigmented epithelioid melanocytic tumor. Therefore, we favor the term "EBN" over the equivocal terms "pigmented epithelioid melanocytoma" or "animal-type melanoma."

\section{Conflicts of Interest}

No potential conflict of interest relevant to this article was reported.

\section{REFERENCES}

1. Carney JA, Ferreiro JA. The epithelioid blue nevus: a multicentric familial tumor with important associations, including cardiac myxoma and psammomatous melanotic schwannoma. Am J Surg Pathol 1996; 20: 259-72.

2. Moreno C, Requena L, Kutzner H, de la Cruz A, Jaqueti G, Yus ES. Epithelioid blue nevus: a rare variant of blue nevus not always associated with the Carney complex. J Cutan Pathol 2000; 27: 218-23.

3. Murali R, McCarthy SW, Scolyer RA. Blue nevi and related lesions: 
a review highlighting atypical and newly described variants, distinguishing features and diagnostic pitfalls. Adv Anat Pathol 2009; 16: 365-82.

4. Granter SR, McKee PH, Calonje E, Mihm MC Jr, Busam K. Melanoma associated with blue nevus and melanoma mimicking cellular blue nevus: a clinicopathologic study of 10 cases on the spectrum of so-called 'malignant blue nevus'. Am J Surg Pathol 2001; 25: 316-23.

5. Busam KJ. Metastatic melanoma to the skin simulating blue nevus. Am J Surg Pathol 1999; 23: 276-82.

6. Zembowicz A, Carney JA, Mihm MC. Pigmented epithelioid melanocytoma: a low-grade melanocytic tumor with metastatic potential indistinguishable from animal-type melanoma and epithelioid blue nevus. Am J Surg Pathol 2004; 28: 31-40.

7. Requena C, Requena L, Sánchez-Yus E, et al. Pigmented epithelioid
Spitz naevus: report of two cases. Histopathology 2006; 49: 549-51.

8. Groben PA, Harvell JD, White WL. Epithelioid blue nevus: neoplasm Sui generis or variation on a theme? Am J Dermatopathol 2000; 22: 473-88.

9. Crowson AN, Magro CM, Mihm MC Jr. Malignant melanoma with prominent pigment synthesis: "animal type" melanoma: a clinical and histological study of six cases with a consideration of other melanocytic neoplasms with prominent pigment synthesis. Hum Pathol 1999; 30: 543-50.

10. Antony FC, Sanclemente G, Shaikh H, Trelles AS, Calonje E. Pigment synthesizing melanoma (so-called animal type melanoma): a clinicopathological study of 14 cases of a poorly known distinctive variant of melanoma. Histopathology 2006; 48: 754-62. 